\title{
Investigating the effect of Anti Stripping Agents (ASAs) on fatigue and rutting properties of Stone Matrix Asphalt (SMA) mixtures modified by Ground Tire Rubber (GTR) and waste polyethylene terephthalate (PET)
}

Alireza Ameli, Javad Maher, Amir Mosavi, Narjes Nabipour, Rezvan Babagoli, Navid Norouzi Oxford Brookes University, Oxford OX30BP, UK; $\underline{\text { a.mosavi@brookes.ac.uk }}$

\begin{abstract}
The current study assessed the influence of Anti Stripping Agents (ASA), Ground Tire Rubber (GTR) and waste polyethylene terephthalate (PET) on performance behavior of binder and Stone Matrix Asphalt (SMA) mixtures. Through this paper the $85 / 100$ penetration grade bitumen was utilized as original bitumen. Also, three liquid ASA's (ASA (A), ASA (B), ASA (C)) were used as mixture modifier. For this purpose, softening point, penetration, rotational viscosity, Dynamic Shear Rheometer, Multi Stress Creep Recovery (MSCR) and Linear Amplitude Sweep (LAS) tests were implemented to investigate the rheological properties of modified bitumen. For evaluating the behavior of modified mixtures several tests such as; Resilient Modulus, Tensile Strength, dynamic creep, wheel track and four point beam fatigue tests were implemented. Based on MSCR test results, utilization of mentioned polymers enhanced the elasticity of bitumens and therefore the permanent deformation resistance of binders increases. Also by addition of PET percentage, the rutting resistance improves. Results indicated that utilization of ASAs, PET and
\end{abstract}


Crumb Rubber (CR) enhance the Resilient Modulus (Mr), Indirect Tensile Strength (ITS), rutting resistance, fatigue life and Fracture Energy (FE) of asphalt mixtures. Also based on results, modification of binder by PET/CR with ratio of 50\%/50\% and ASA (B) have the highest fatigue life which indicates that this mixture have highest resistance against fatigue cracking.

Keywords: Ground Tire Rubber (GTR); Anti Stripping Agents (ASA); Stone matrix asphalt (SMA); waste polyethylene terephthalate (PET); Rutting; Fatigue.

\section{Introduction}

All around the world several waste industrial materials are produced. These vast volume of materials have negative influence on environment such as: soil, air and water pollution, that influence the economic issues, human life, energy preservation. For this reason lots of attention was attracted to reuse of mentioned materials as alternatives and reduce consumption of new sources. occupation of vast area by these materials is another drawbacks of waste materials. So further investigations was needed on reusing of waste materials in pavement industry [1-5].

One of the main kinds of plastics is Polyethylene Terephthalate (PET) [6]. This kind of plastics compound of high ratio of thermoplastic polymers. This waste material is a semi-crystalline thermoplastic polymer and is kind of polyester solid [7]. Large amount of waste PET is being produced in the form of different products, for instance, bottles, fibers, molding and sheets are the products mostly manufactured in Europe by application of PET [8]. Utilization of PET is well-known in food industry because of superlative characteristics offered by PET as a packaging material, mainly as bottles $[9,10]$.

One of the most important asphalt modifiers which is used for many years in pavement industry 
and reclaimed from vehicle and also truck tires is Ground Tire Rubber (GTR). The GTR is normally added to the bitumen at a range of $15-20 \%$, according to the source and PG of original binder. The utilization of GTR with polymer modified binder cause an enhancement of performance of binder[11]. For many years utilization of GTR in asphalt mixture was evaluated as an convenient material to enhance original mixture properties and removal of crumb tires [12]. Several researches in the past indicated that addition of crumb rubber as a thermoplastic elastomer to original bitumen led to enhance its properties against fatigue and rutting, decrease the rehabilitation and maintenance prices of mixture, improved pavement life, reduced traffic noise [13-15].

A good modifier should enhance the performance of bitumen against series of failures. Based on a literature one modifier is incapable of improving all performances of pavements. Therefore, modification of bitumen with more than one modifier is essential, which may obtain multiple performance improvements due to multiple interaction [16 ].

In a study performed by Wang, Wang, Wu, and Zhang [17], the influence of Polyethylene Terephthalate which is composition of polyethylene and crumb rubber on rheological binder. Results revealed that utilization of mentioned materials could enhance storage modulus of modified binder.

Nazirizad [19] evaluated the influence of ASA and hydrated lime on water sensitivity of samples. The results showed that mixtures modified by ASA have better resistance against moisture than mixture modified by hydrated lime. Park et al. [20] evaluated that aliphatic amine ASA could enhance the rutting behavior and moisture damage resistance of pavement. Zheng et al. [21] revealed that it is necessary to investigate the adaptability between ASA and bitumen 
before selection of ASA. Xiao et al. [22] investigated the impact of ASA additive on stripping and permanent deformation properties of pvements. Results indicated that ASA cause a little improvement on rutting performance of mixtures, but used ASAs are incapable of changing the PG of original binder. Also Selvaratnam et al. [23] indicated that ASA with different contents led to better change PG of PG 70-28 and PG 76-28 polymer modified bitumens in comparison with PG 64-22.

Several studies were implemented on using discarded PET in mixtures by replacing with coarse and fine aggregate of asphalt mixture and also for modification of different types of mixtures. By replacing PET materials by coarse aggregates it led to improve the properties of mixture such as Marshall Stability and Marshall Quotient [23]. Also by replacing PET by fine aggregate in mixtures it cause an increase in rutting resistance of mixture and decrease the stiffness of mixture [24]. Ahmadinia investigated the usage of waste PET in dry method in SMA mixtures. The results showed that addition of PET led to enhance the Stability and behavior of SMA specimens [25]. Also several studies revealed that addition of PET led to enhance permanent deformation, fatigue and moisture vulnerability resistance of SMA mixtures [10, 18, 33-35].

Based on a literature one modifier is incapable of improving all performances of pavements. Therefore, modification of bitumen with more than one modifier is essential, which may obtain multiple performance improvements due to multiple interaction [16 ].

Far too little research studied on the enhancement in rheological properties of binder and performance of CR/PET modified mixtures. As usual ASAs have indicated an enhancement on water susceptibility and performance of unmodified samples, the feasibility of application of ASAs on PET/CR modified mixtures should be investigated [24]. therefore, it is essential to 
investigate the performance of PET/CR SMA mixtures. In current work, PET/ground tire rubber (GTR) composites with different composition were utilized for modification of bitumen and enhance its performance. The influence of three ASA on PET/CR modified binders were evaluated through series of physical and rheological tests including; MSCR, LAS, rotational viscosity, softening point, penetration grade, dynamic shear rheometer (DSR) tests. The four point beam fatigue, wheel track, dynamic creep, Resilient Modulus, Indirect Tensile Strength (ITS) test were implemented to investigate the performance of mixtures.

\section{Materials and methods}

\subsection{Materials used}

\subsubsection{Aggregates}

The required aggregates were supplied from Telo Quarry. The aggregate's physical and chemical properties were shown on Table 1 and 2, respectively. In this study the nominal maximum aggregate size of $12.5 \mathrm{~mm}$ was utilized and it's gradation was depicted on Figure 1.

\subsection{2. bitumen}

One type of virgin binder AC-85/100 was utilized and the bitumen's properties were tabulated on Table 3.

\subsection{3. fiber}

As the National Cooperative Highway Research Pavement (NCHRP) Report No. 425 [30] suggested, by utilizing $0.3 \%$ cellulose fiber in dry process to the mixture, the drain down of 
binder will not be occur. Table 4 tabulated the properties of fiber.

\subsubsection{Polymers (PET, Crumb rubber)}

The size of GTR is -40 mesh fabricated by an ambient process. Table 5 shows the properties of crumb rubber. The waste plastic bottles were collected to utilize as an additive. At first, the mentioned waste materials were cleaned, dried, cut into smaller parts and added in different percentage $(25 \%, 50 \%$ and $75 \%$ by weight of aggregates) to the mixture. The properties of PET was shown on Table 6. Based on Table 8, three different blends of PET and CR with constant content of water were combined. Based on previous literature by considering water At high pressure and temperature, the PET depolymerized into its monomers (terephthalic acid) because of acting water as hydrolysis agent [31]. Each blend was separately passed through a twin-screw extruder with the speed of $65 \mathrm{rpm}$ at the temperature of $280^{\circ} \mathrm{C}$. Ultimately, in order to better mix the combination in high shear mixer the products were granulated.

\subsubsection{ASAs}

Three usual liquid ASAs, namely liquid A, liquid B and liquid C were selected.

The liquid ASAs are typically used in percentages between 0.2 and $0.6 \%$ by weight of the bitumen based on manufacture's recommendations. In this research the fix percentage of $0.5 \%$ was used for all mixtures. Table 7 tabulates the Physical and chemical properties of used ASAs. 


\subsection{Sample Preparation}

Crumb rubber modification process of original binder was in accordance of wet process. At first, 800 gr of original binder was warmed up to $170{ }^{\circ} \mathrm{C}$ in a steel bowl to reach in liquid form. After that the additives was added gradually at $15 \%$ (three blends of PET and CR $(25 \%, 50 \%$ and $75 \%$ ) by weight of original bitumen) to the high shear mixer and mixed at $500 \mathrm{rpm}$. After that the temperature rose up to $190^{\circ} \mathrm{C}$, and also the speed of high shear mixer increased up to 4000 rpm for $2 \mathrm{~h}$. At last, in order to eliminate air bubbles which were produced in high shear mixer in producing procedure, the specimens were located in a vacuum oven for $30 \mathrm{~min}$ at $120^{\circ} \mathrm{C}$. It is suggested by previous papers to blend ASAs with bitumen instead of adding them to mixture [31]. So three ASAs with constant percentage of $0.5 \%$ by weight of binder were utilized into CR/PET modified bitumen and mixed by high shear mixer at $1000 \mathrm{rpm}$ for $45 \mathrm{~min}$. Several samples with different ASA were fabricated and sample identification of modified binders were shown on table 8 .

The NCHRP Report No. 425 was used to design and fabricate the SMA mixtures [30]. According to volumetric properties of mixtures the optimum bitumen percentage was calculated as $7.5 \%$. For each mixture type, three specimens were prepare.

\section{Experimental Programme}

\subsection{Binder tests}

\subsubsection{Conventional and Rheological binder tests}

In present research the penetration grade, softening point, ductility tests were implemented in order to measure the physical properties of binders.

\subsubsection{MSCR test}


In current work, to evaluate the rutting resistance of binders the MSCR test was performed through AASHTO TP 70 standard [31].

\subsubsection{LAS test}

In current work to investigate the fatigue resistance of binders, the LAS test was performed according to AASHTO TP 101-14 standard [32]. The fatigue life of bitumens were obtained based on equation (1):

$$
\mathrm{Nf}=\mathrm{A}\left(\gamma_{\max }\right)^{\mathrm{B}}
$$

where $A$ and $B$ are coefficients of equation measured based viscoelastic continuum damage theory (VECD).

\subsection{Mixture tests}

\subsubsection{ITS test}

The main properties of mixture is tensile strength which is measured by ITS test. In most cases, the ITS test was implemented to investigate the water damage of pavements. According to ASTM D6931-12 standard, the ITS test was performed at $20^{\circ} \mathrm{C}$. The following equation was utilized to determine and compare the ITS of specimens.

$$
I T S=2 P_{\max } / \pi D t
$$

where,

ITS refers to the indirect tensile strength of mixture $(\mathrm{kPa}), \mathrm{P}_{\max }$ indicates the maximum load 
$(\mathrm{kN})$; D shows diameter of the samples $(\mathrm{mm})$; $\mathrm{t}$ refers to thickness of the specimens $(\mathrm{mm})$.

\subsection{2. $M_{r}$ test}

The $M_{r}$ of mixtures was calculated based on ASTM D 4123. All mixtures were divided in to two set. One set was remained dry at $25^{\circ} \mathrm{C}$, and second set of samples was emerged in water based on AASTHO T283 and referred to as conditioned samples. Then the Mr test was run at $25^{\circ} \mathrm{C}$ by applying haversine load pulse at $1 \mathrm{~Hz}$ with 0.1 -s loading and unloading time, respectively. At last RMR parameter which was referred to the ratio of $M_{r}$ of wet samples to the $M_{r}$ of dry specimens, were measured. The minimum adequate value of $80 \%$ was considered for RMR results[33]:

Where,

$$
M r=P(v+0.2734){ }_{\delta t}
$$

$P$ refers to maximum load applied $(\mathrm{N})$;

$v=$ Poisson's ratio;

$\mathrm{t}=$ length of sample $(\mathrm{mm})$;

$\delta=$ horizontal recoverable deformation $(\mathrm{mm})$.

\subsubsection{Dynamic creep test}

In current work the US.NCHRP 9-19 standard was utilized to investigate the strength of samples versus rutting through dynamic creep test. The samples were maintained on chamber with a controlled temperature of $50{ }^{\circ} \mathrm{C}$. The stress level of $450 \mathrm{KPa}$ with $0.1 \mathrm{~s}$ loading and $0.9 \mathrm{~s}$ unloading time was used to investigate the rutting behavior of mixtures.

\subsubsection{Wheel tracking test}


To measure the permanent deformation potential of SMAs the wheel track test was implemented according to AASHTO Standard T-324 [34] at $60^{\circ} \mathrm{C}$ temperature.

\subsubsection{FPB test}

for investigating the intermediate temperature behavior of specimens the FPB test was implemented based on AASHTO T321-07. Following equations were used to measure the flexural stiffness [35]:

$$
\begin{aligned}
& =\begin{array}{lll}
12 & h & 10^{6}
\end{array} / 3\left(\begin{array}{ll}
G_{0}^{2} & \left.4 G_{1}^{2}\right)
\end{array}\right. \\
& =G_{0} P / B h^{2} \\
& S=1000
\end{aligned}
$$

Where,

$\varepsilon$ refer to maximum micro-strain;

$\delta$ refer to maximum displacement at the middle of the beam $(\mathrm{mm})$;

$h$ shows length of beam (mm);

$G_{0}$ indicates outer $(355.5 \mathrm{~mm})$;

$G_{1}$ indicates the inner length of the gauge $(118.5 \mathrm{~mm})$;

$\sigma$ shows maximum tensile stress $(\mathrm{kPa})$;

$P$ refer to maximum load $(\mathrm{kN})$;

$B$ refer to beam width $(\mathrm{mm})$ 
$S$ refer to flexural stiffness (MPa).

Equation (7) was used to evaluate the fatigue life of the samples:

$$
N_{f}=a \varepsilon^{-b}
$$

Where,

$N_{f}$ is fatigue life of specimens;

$\varepsilon$ is applied levels of micro strains;

$a$ and $b$ are coefficients.

\subsubsection{Fracture energy}

The fracture energy to failure of samples for both dry and unconditioned type were determined from ITS test to investigate the water sensitivity of samples. The fracture energy to failure is defined as the area under the load- deflection curve at failure load. (Figure 2) and can be determined by equation (8) [36]:

$$
\text { Fracture energy }=\frac{\int_{0}^{\delta \max } P(\delta) d(\delta)}{H D}
$$

Where,

fracture energy refer to total fracture energy at failure $\left(\mathrm{J} / \mathrm{m}^{2}\right)$,

$\mathrm{P}$ indicate load $(\mathrm{N})$,

$\mathrm{d}$ refer to deformation $(\mathrm{mm})$, 
$\mathrm{H}$ refer to the diameter of sample $(\mathrm{mm})$ and,

$\mathrm{D}$ refer to the diameter of sample $(\mathrm{mm})$.

\section{Results}

\subsection{Bitumen test results}

\subsubsection{Rheological and conventional bitumen test result}

Figures 3-5 show the outcomes of physical binder tests of virgin and modified bitumens.

According to results, addition of PET and CR to virgin bitumen cause an improve the properties of bitumen. Based on figures 3,4, addition of PET and CR decreases and increases the penetration grade and softening point of bitumen. The reason for this would be due to stiffening of binder by addition of that PET and CR. As results indicate, by increasing in ratio of PET/rubber, the binder becomes stiffer and as a result it led to decreases the penetration and increases the softening point of binder. As the percentage of ASA (A) increase the penetration had a reduction trend and softening point had increasing trend. Also results indicated that by addition of ASAs the penetration values of PER/CR modified binders decreases. Among ASAs additive ASA (B) have better effect to reduce the penetration of binders. Also it can be seen from the results that ASA $(\mathrm{C})$ had lower effect on reducing the penetration of binders. Based on results, It can be find out that utilization of additives make the bitumen stiffer and as a result the resistance of binder against rutting improves. Based on the results addition of ASAs led to increase the softening point of samples. Among ASAs additive ASA (B) have better effect to increase the softening point of binders.

As demonstrated on figure 5, using PET/CR cause an increase in viscosity of virgin bitumen. As the viscosity of binder increases, the ability to lay down and compaction of mixture on field 
becomes harder. According to viscosity of bitumens, as the ratio of PET/rubber increases, the stiffness of bitumen increases and it leads to enhance the viscosity of bitumen. Viscosity test results indicated that by addition of ASAs additives, the viscosity of PET/CR modified bitumen increases. Also according to the results utilization of ASA (A) additive had greater impact on increasing the viscosity of binders followed by ASA (B) and ASA (C).

Binders with higher $\mathrm{G}^{*} / \mathrm{sin} \delta$ values, have higher resistance a better resistance against permanent deformation. Original and RTFO aged binders should have a minimum value of $1 \mathrm{kPa}$ and 2.2 $\mathrm{kPa}$ to be resist against rutting. Results of rutting parameter $\left(\mathrm{G}^{*} / \sin \delta\right)$ was shown on Figure 5 . Based on results, the rutting resistance of binders modified by PET/CR is enhanced, which demonstrates that utilization of PET/CR make binder more stiffer and then the rutting behavior of binder improves. Based on the results, as the ratio of PET/CR increases, the stiffness of binder increases, so the permanent deformation resistance of binders increases.

Results from figure 6 indicates that addition of ASAs irrespective of ASA type led to increase the rutting parameter. It is attributed to stiffening of binder by addition of ASA's. Among ASAs, the binder modified by ASA (B) have higher rutting parameter which is due to the formation of organogels after utilization of ASAs in PET/CR modified binders. In addition, based on the results illustrated in figure 6 (b), after RTFO aging process, the $G^{*} /$ sin d values increased in comparison to unaged samples. This is due to the fact that the light components of binder vaporize and the aromatics and resins change to the asphaltenes in bitumen[37-38].

Results indicated that all ASAs improve the rutting performance of PET/CR modified binders after RTFO aging. Moreover, for ASA (B) the PET/CR with $0.75 \%$ PET and 0.25 CR indicates the highest rutting parameter. 


\subsubsection{MSCR test}

In this research to evaluate the rutting behavior of bitumen at two stress levels of $100 \mathrm{~Pa}$ and $3200 \mathrm{~Pa}$ the MSCR test was performed. The Jnr parameter of bitumens at temperatures of $52{ }^{\circ} \mathrm{C}$ to $82^{\circ} \mathrm{C}$ at mentioned stress levels were depicted on figure 7 . Results indicated that as the temperature increases, the jnr parameter and percent recovery increase and decrease, respectively. Table 9 shows the non-recoverable creep compliance and recovery percentage of binders at $64{ }^{\circ} \mathrm{C}$. Generally, based on the results regardless of the stress levels, the addition of PET/CR to base bitumen decreased the non-recoverable creep compliance value of bitumen which led to enhance the rutting performance of original binder. As results show on table 9, by addition of ASA's the Jnr values decreased. Also between the ASA's used in this study the ASA (A) had the best performance on enhancing the rutting behavior of bitumens. According to outcomes, ASA's stiffens the bitumen and the strength of binders against rutting increased. By addition of stress level to $3.2 \mathrm{kpa}$, the Jnr values increased. It means that higher permanent deformation would be observed on higher traffic loading. The similar trend observed on Jnr values of modified binders on stress level of $3.2 \mathrm{Kpa}$.

As PET/CR additive added to the binder, the recovery percentage increases. Results on table 9 indicates that the percent recovery percentage of base bitumen is low which shows its low ability against intermediate temperature cracking and rutting. Based on table 9, bitumens modified by ASA's have greater percent recovery values. as the percentages of ASA's increases, the percent recovery increases which indicates that the strength of binders versus permanent deformation was enhanced. According to the results, among ASA's, the ASA (B) specimen has the highest recovery which show the most enhancement of rutting behavior of binder. Results show that utilization of three ASA with different percentages is capable of enhancement of rutting 
performance of PET/CR modified binders, which is in the same manner with the results from $\mathrm{G}^{*} / \operatorname{Sin} \delta$.

utilization of PET/CR in original bitumen enhances the recovery percentage by $42 \%$ and $161 \%$, respectively, for 100 pa stress level.

As results show, the ASA (C) additive had lowest effect on enhancing percent recovery of modified binders for stress levels of 100 and 3200 pa. This could be due to lower elasticity and of ASA modified binders.

\subsubsection{LAS results}

The results of LAS test was depicted on tables 10, 11. Results from Table 10 shows that in high strain levels the shear stress of modified binders decreases significantly which indicates a high level of damage. Results indicated that utilization of PET/CR cause an enhancement in fatigue life of original binder. utilization of PET/CR increase the flexibility of binder. So the fatigue behavior of bitumen increases.

The VECD coefficients of different modified binders was shown in Table 10. utilization of modifiers cause an increase and decrease in coefficient $\mathrm{C} 1$ and coefficient $\mathrm{C} 2$, respectively. Based on the results, PET/CR modified bitumen containing 50\% PET and 50\% CR has the highest $\mathrm{C} 1$ and the lowest $\mathrm{C} 2$ values. it can be concluded that at low damage levels the $\left|\mathrm{G}^{*}\right|$ 
parameter decreases significantly adversely at high damage levels the $\left|\mathrm{G}^{*}\right|$ parameter reduces lower.

Table 11 shows the fatigue life of different bitumens. The fatigue test was performed on two strain levels of $2.5 \%$ and $5 \%$. As results show, utilization of PET/CR cause an enhance the fatigue life of virgin binder. In binders modified by PET/CR, the combination of 50\% PET and $50 \% \mathrm{CR}$ have the highest fatigue life. Also addition of ASA's cause an improvement on fatigue life of binders. Binders containing ASAs have the same trend like PET/CR modified binders. Based on the results among ASA's modified binder the ASA (B) have the most influence on improvement of fatigue performance of binders. Based on the results, by addition of strain rate, the fatigue life of binders decreases. From the results it is apparent that PC2, PC2B modified binders has higher fatigue life about 1.77 times and 2.31 times more than original bitumen at both strain levels of $2.5 \%$ and $5 \%$, respectively.

\subsubsection{Grading of binders based on PG and MSCR system}

In current work, the PG and MSCR method are used in order to grade of modified. Results are depicted on Table 12. The $\mathrm{G}^{*} / \mathrm{Sin} \delta$ parameter is limited to $1 \mathrm{kPa}$ to grade of non-aged binder, while for RTFO aged binder the MSCR method in standard traffic ( $\mathrm{Jr}-3.2 \mathrm{kPa}=4.5 \mathrm{kPa}-1)$. To evaluate and grade of bitumens at low temperatures, the same criteria like PG system was used.

As results indicated in Table 12, all modified binders indicate one high temperature grade improvement, while in the $75 \% \mathrm{PET}+25 \% \mathrm{CR}$ modified by ASA (B) indicate two high temperature grade improvement (PG 70-16) of original binder by PG 58-16. However, all 
PET/CR modified binders and binders containing ASA (A) (except PC3A) indicate one low temperature improvement.

As results show, almost all modified bitumens are graded the same high-temperature and also one grade improvement at a standard traffic level through PG and MSCR system.

\subsection{Mixture tests result}

\subsubsection{Mr test results}

The Mr test results of specimens modified by different additives were shown on Figure 8. Based on the Results by utilization of PET/CR, the Mr values enhance. Based on the results the Mr values of modified samples are better than original mixture. By addition of PET/CR ratio the increasing trend was observed up to $50 \%$ PET and 50\% CR. While by utilization of higher PET content cause the Mr values to decrease. Also addition of ASAs cause an enhance the Mr of mixtures. So that the PC2B mixture has the highest Mr value among specimens containing ASA. The reason for this may attributes to stiffen of binder with ASA. Based on figure $8, \mathrm{Mr}$ values of mixtures modified by ASA (A) and (C) have increasing trend up to 50\% PET, however utilization of more percentages cause the Mr values to decrease.

The Mr of PC2B sample was $15 \%$ higher than original mixture, however PC $3 \mathrm{C}$ specimen had a Mr value approximately $1 \%$ higher than original sample.

\subsubsection{ITS test}

In order to investigate the effect of different modifiers on tensile behavior of mixtures, the ITS test was performed. Figure 9 shows the ITS results of samples. Outcomes of ITS test revealed 
that specimens modified by PET and CR additive had greater ITS values than control mixture. By addition of ratio of PET in composition, the ITS results first increases up to 50\%, and then decreases up to $75 \%$ PET. By addition of ASA to PET/CR modified mixtures, the ITS values increased. Among modified mixtures, PC2B mixture has the highest ITS value. Also ITS of specimens modified by ASA (B) are higher than ITS values of mixtures modified by ASA (A) and $(\mathrm{C})$.

As the cohesion of bitumen to aggregate improves, the ITS values becomes higher [39-42]. It is recognized from the ITS values that utilization of PET/CR and ASA cause to increase and decreases the cohesion and adhesion of binder to aggregate, respectively.

\subsubsection{Flow Number (FN) results}

Figure 10 depicts the FN of modified specimens. mixtures with greater FN are capable of resisting better against permanent deformation. [43-46]. As results show on figure 10, utilization of PET/CR enhance the permanent deformation resistance of samples. This could be due to the fact that by utilization of PET and CR the flexibility of specimens increases and the ability of mixtures to recover their accumulated strain was improved. Also addition of ASA's cause the FN of mixtures to increase. Based on results, it can be recognized that addition of ASA cause the stiffness and viscosity of mixtures to increase and therefore the permanent deformation performance enhances. As results show, utilization of PET/CR additive led to increase the flow number of specimens by $29 \%$. Also addition of ASA (A), (B) to $75 \% \mathrm{PET}+25 \% \mathrm{CR}$ cause an increase in FN of PET/CR modified mixture by $11 \%, 31 \%$ respectively. While addition of ASA (C) cause an increase in FN values of PET/CR modified mixture by $6 \%$.

\subsubsection{Results of wheel tracking test}


Figure 11 shows the rut depth of specimens. As results show, PET/CR cause the rut depth of mixtures to decrease. PET and CR improves the flexibility of mixtures. Results indicated that as the percentages of PET improves the permanent deformation performance of mixtures. Also addition of ASA enhanced the rutting behavior of mixtures. Among ASAs, ASA (B) has better effect on improving the permanent deformation performance of mixtures. As results show, addition of combination of $75 \%$ PET and $25 \%$ CR additive to base bitumen cause an decrease the rut depth of specimens by $27 \%$.

\subsubsection{Four point beam fatigue test}

Figure 12 shows fatigue behavior of specimens. results demonstrated that utilization of PET/CR improve the fatigue behavior of specimens. so that all modified mixtures have higher fatigue life than control mixture. By addition of PET percentages up to $50 \%$, the ascending trend is observed, while addition of $75 \%$ PET have decreasing trend. It might be due to the fact that the CR powder enhance the detrimental properties of PET as plastic component. It can be seen from the results that addition of ASA's cause an improvement in fatigue performance of specimens. among ASAs added to the mixture, ASA (B) have better ability to improve the fatigue life of mixtures. Mixtures containing ASAs have the same trend like mixtures without ASA (PC1, PC2, PC3).

\subsubsection{FE test results}

The Fracture energy density of different specimens were shown on figure 13 . The required energy to initiate the first crack in asphalt called Fracture Energy (FE). Based on Figure 13, utilization of PET/CR result in enhance the fracture energy. This can be due to increase in elasticity of specimens by using PET/CR and consequently the strain energy and strength of 
specimen to cracking is increased. Addition of PET up to 50\% led to increase in fracture energy of specimens and by addition of more percentages, the fracture energy decreases. It can be concluded that by addition of 50\% PET and 50\% CR strength of mixture versus intermediate temperature cracking enhances and by addition of more PET content in composition the fatigue cracking decreases. Addition of ASAs cause an increase the FE. Among mixtures modified by ASAs the ASA (B) has the highest impact on increasing the FE of mixtures. PC2B mixture has the highest FE among modified mixtures.

\section{Discussions and Conclusion}

The goal of current research was to evaluate the effect of PET/CR and three different ASA on rheological performance of bitumen and performance properties of SMA mixtures. Hence, several rheological and performance tests were performed such as; MSCR, LAS, ITS, MR, dynamic creep, wheel track and fatigue life were implemented. The following conclusions can be derived from the results:

- Based on MSCR test results, regardless of the stress levels, the addition of PET/CR to base bitumen decreased the non-recoverable creep compliance value of bitumen which led to enhance the rutting performance of original binder. Also by addition of ASA's the Jnr values decreased.

- Based on LAS test results, utilization of PET/CR cause an enhance the fatigue life of virgin binder. In binders modified by PET/CR , the combination of 50\% PET and 50\% CR have the highest fatigue life. Also addition of ASA's cause an improvement on fatigue life of binders.

- Based on ITS test results, ITS values of mixtures containing PET and CR additive were greater than control mixture. By addition of ASA to PET/CR modified mixtures, the ITS 
values increased. Among modified mixtures, PC2B mixture has the highest ITS value.

- According to the Mr values of mixtures, utilizing of PET/CR cause the resilient modulus to increase. PET/CR modified mixtures have $30 \%$ higher resilient modulus than unmodified mixtures. This increment attributed to increase in flexibility of mixtures by addition of PET and CR. Also addition of ASAs cause an enhance the Mr of mixtures. So that the PC2B mixture has the greatest Mr value among samples modified by ASA.

- Based on FE results of mixtures, utilizing of PET/CR cause an enhance the fracture energy. This can be due to increase in flexibility of specimen by utilizing PET/CR and consequently the strain energy and strength of specimen to cracking is increased. Addition of ASAs cause an increase the FE. Among mixtures modified by ASAs the ASA (B) has the highest impact on increasing the FE of mixtures. PC2B mixture has the highest FE among modified mixtures.

- Based on dynamic creep test, using PET/CR enhance the permanent deformation properties of samples. utilization of ASA's cause an enhance the FN of specimens. According to the results, by addition of PET/CR ratio, the rutting resistance improves. It is recognized that utilization of ASA cause an enhance the stiffness and viscosity of mixtures and as a result the rutting behavior enhanced.

- Based on wheel track test results, addition of PET/CR enhanced the permanent deformation behavior of mixtures and by increase of PET/CR ratio, the rutting resistance improves. As results show, addition of ASAs to base bitumen cause an enhance the rutting resistance of specimens.

- According to the four point beam fatigue test utilization of PET/CR enhance the fatigue resistance of specimens. results revealed that utilization of ASA's cause an increase in fatigue performance of specimens. addition of PET/CR enhances the elasticity behavior 
of mixture and as a result, the fatigue life improves.

\section{References}

[1] Rezvan, B., \& Hassan, Z. (2017). Evaluation of rutting performance of stone matrix asphalt mixtures containing warm mix additives. Journal of Central South University, 24(2), 360-373.

[2] Babagoli, R., Hasaninia, M., \& Mohammad Namazi, N. (2015). Laboratory evaluation of the effect of Gilsonite on the performance of stone matrix asphalt mixtures. Road Materials and Pavement Design, 16(4), 889-906.

[3] Xue, Y., Hou, H., Zhu, S., \& Zha, J. (2009). Utilization of municipal solid waste incineration ash in stone mastic asphalt mixture: pavement performance and environmental impact. Construction and Building Materials, 23(2), 989-996.

[4] Fontes, L. P., Triches, G., Pais, J. C., \& Pereira, P. A. (2010). Evaluating permanent deformation in asphalt rubber mixtures. Construction and Building Materials, 24(7), 1193-1200.

[5] Ismail, Z. Z., \& Al-Hashmi, E. A. (2008). Use of waste plastic in concrete mixture as aggregate replacement. Waste Management, 28(11), 2041-2047.

[6] Li, Y., White, D. J., \& Peyton, R. L. (1998). Composite material from fly ash and postconsumer PET. Resources, conservation and recycling, 24(2), 87-93.

[7] Shukla, S. R., \& Harad, A. M. (2006). Aminolysis of polyethylene terephthalate waste. Polymer degradation and stability, 91(8), 1850-1854.

[8] Torres, N., Robin, J. J., \& Boutevin, B. (2000). Study of thermal and mechanical properties 
of virgin and recycled poly (ethylene terephthalate) before and after injection molding. European Polymer Journal, 36(10), 2075-2080.

[9] Navarro, R., Ferrandiz, S., Lopez, J., \& Segui, V. J. (2008). The influence of polyethylene in the mechanical recycling of polyethylene terephtalate. Journal of Materials Processing Technology, 195(1), 110-116.

[10] Moghaddam, T. B., Soltani, M., \& Karim, M. R. (2014). Evaluation of permanent deformation characteristics of unmodified and Polyethylene Terephthalate modified asphalt mixtures using dynamic creep test. Materials \& Design, 53, 317-324.

[11] Lo Presti D, Airey G. Tyre rubber-modified bitumens development: the effect of varying processing conditions. Road Mater Pavement Des 2013;14(4):888-900.

[12] H. H. Kim, K. D. Jeong, M. S. Lee, S. J. Lee, Performance properties of CRM binders with wax warm additives, Constr. Buil. Mater. 66 (2014) 356-360.

[13] H. H. Kim, S. J. Lee, Effect of crumb rubber on viscosity of rubberized asphalt binders containing wax additives, Constr. Buil. Mater. 95 (2015) 65-73

[14] H. H. Kim, M. Mazumder, S. J. Lee, Recycling of aged asphalt binders with wax warm additives, Road Mater. Pave. Des. 19 (5) (2018) 1203-1215.

[15] Y. Yan, R. Roque, D. Hernando, S. Chun, Cracking performance characterisation of asphalt mixtures containing reclaimed asphalt pavement with hybrid binder, Road Mater. Pave. Des. 20 (2) (2019) 347-366.

[16] Ameli, A., Babagoli, R., \& Aghapour, M. (2016). Laboratory evaluation of the effect of 
reclaimed asphalt pavement on rutting performance of rubberized asphalt mixtures. Petroleum Science and Technology, 34(5), 449-453.

[17] Wang, S., Wang, Q., Wu, X., \& Zhang, Y. (2015). Asphalt modified by thermoplastic elastomer based on recycled rubber. Construction and Building Materials, 93, 678-684.

[18] F. Xiao, S.N. Amirkhanian, Effects of liquid antistrip additives on rheology and moisture susceptibility of water bearing warm mixtures, Constr. Build. Mater. 24 (2010) 1649-1655.

[19] M. Nazirizad, A. Kavussi, A. Abdi, Evaluation of the effects of anti-stripping agents on the performance of asphalt mixtures, Constr. Build. Mater. 84 (2015) 348-353.

[20] D.W. Park, W.J. Seo, J. Kim, H.V. Vo, Evaluation of moisture susceptibility of asphalt mixture using liquid anti-stripping agents, Constr. Build. Mater. 144 (2017) 399-405.

[21] C.F. Zheng, Y. Qin, D. Lv, T. Zhang, X.Y. Liu, S. Zheng, Effects of anti-stripping agents on the microscopic strength of mineral aggregate contact surface, Constr. Build. Mater. 49 (2013) $627-634$.

[22] F. Xiao, S.N. Amirkhanian, Effects of liquid antistrip additives on rheology and moisture susceptibility of water bearing warm mixtures, Constr. Build. Mater. 24 (9) (2010) 1649-1655.

[23] S. Selvaratnam, M. Zaman, G. Laguros, Laboratory evaluation of unmodified and polymermodified performance-grade binders with anti-stripping additives, J. Eng. Comput. Archit. Sci. J. Int. (SJI) 1 (2) (2007) 1934

[24] Hassani, A., Ganjidoust, H., \& Maghanaki, A. A. (2005). Use of plastic waste (polyethylene terephthalate) in asphalt concrete mixture as aggregate replacement. Waste 
Management \& Research, 23(4), 322-327.

[25] Rahman, W. M. N. W. A., \& Wahab, A. F. A. (2013). Green pavement using recycled polyethylene terephthalate (PET) as partial fine aggregate replacement in modified asphalt. Procedia Engineering, 53, 124-128.

[26] Ahmadinia, E., Zargar, M., Karim, M. R., Abdelaziz, M., \& Shafigh, P. (2011). Using waste plastic bottles as additive for stone mastic asphalt. Materials \& Design, 32(10), 4844-4849.

[27] Ahmadinia, E., Zargar, M., Karim, M. R., Abdelaziz, M., \& Ahmadinia, E. (2012).

Performance evalua- tion of utilization of waste polyethylene terephthalate (PET) in stone mastic asphalt. Construction and Building Materials, 36, 984-989.

[28] Moghaddam, T. B., Karim, M. R., \& Syammaun, T. (2012). Dynamic properties of stone mastic asphalt mixtures containing waste plastic bottles. Construction and Building Materials, $34,236-242$.

[29] Moghaddam, T. B., Soltani, M., \& Karim, M. R. (2014b). Experimental characterization of rutting per- formance of polyethylene terephthalate modified asphalt mixtures under static and dynamic loads. Construction and Building Materials, 65, 487-494.

[30] Brown ER, Cooley LA. Designing stone matrix asphalt mixtures for rut-resistant pavements. Transport Res Board 1999.

[31] AASHTO. 2010. Standard practice for multiple stress creep recovery test of asphalt binder using a dynamic shear rheometer. AASHTO TP70. Washington, DC: AASHTO.

[32] AASHTO, 2006. Standard specification for transportation materials and methods of 
sampling and testing. American Association of State Highway and Transportation Officials.

[33] Campanelli, J. R., Kamal, M., \& Cooper, D. (1993). A kinetic study of the hydrolytic degradation of polyethylene terephthalate at high temperatures. Journal of Applied Polymer Science, 48(3), 443-451.

[34] AASHTO, T. (2013). 324, Hamburg Wheel-Track Testing of compacted Hot Mix Asphalt (HMA).

[35] Ziari, H., Babagoli, R., \& Akbari, A. (2015). Investigation of fatigue and rutting performance of hot mix asphalt mixtures prepared by bentonite-modified bitumen. Road Materials and Pavement Design, 16(1), 101-118.

[36] Ziari H, Babagoli R, Ameri M, Akbari A. Evaluation of fatigue behavior of hot mix asphalt mixtures prepared by bentonite modified bitumen. Construction and Building Materials. 2014 Oct 15;68:685-91.

[37] Ziari, H., Babagoli, R., \& Razi, S. E. T. (2015). The evaluation of rheofalt as a warm mix asphalt additive on the properties of asphalt binder. Petroleum Science and Technology, 33(2122), 1781-1786.

[38] S.C. Xu (Ed.), Organic Chemistry, Higher Education Press, Beijing, 2014, pp. 377-378 (in Chinese).

[39] R.D. Mahapatra, J. Dey, Instant gels from mixtures of amines and anhydrides at room temperature, Colloids Surf. B 147 (2016) 422-433.

[40] Hassan, Z., Hassan, D., Rezvan, B., \& Ali, A. (2012). Influence of bentonite additive on 
bitumen and asphalt mixture properties. World Academy of Science, Engineering and Technology, 6 .

[41] Norouzi, N., Ameli, A., \& Babagoli, R. (2019). Investigation of fatigue behaviour of warm modified binders and warm-stone matrix asphalt (WSMA) mixtures through binder and mixture tests. International Journal of Pavement Engineering, 1-10.

[42] Muftah, A., Bahadori, A., Bayomy, F., \& Kassem, E. (2017). Fiber-Reinforced Hot-Mix Asphalt: Idaho Case Study. Transportation Research Record, 2633(1), 98-107.

[43] Bayomy, F., Muftah, A., Wen, H., \& Bahadori, A. (2016). Evaluation of Fiber-Reinforced Asphalt Pavements: Laboratory Study (No. FHWA-ID-16-237). Idaho. Transportation Dept..

[44] Zhang, W., Bahadori, A., Shen, S., Wu, S., Muhunthan, B., \& Mohammad, L. (2018). Comparison of Laboratory and Field Asphalt Aging for Polymer-Modified and Warm-Mix Asphalt Binders. Journal of Materials in Civil Engineering, 30(7), 04018150.

[45] Zhang, F. and Hu, C., 2015. Physical and rheological properties of crumb rubber/styreneebutadieneestyrene compound modified asphalts. Polymer Composites. doi:10.1002/pc.23762.

[46] Leng, Z., Padhan, R.K., and Sreeram, A., 2018a. Production of a sustain- able paving material through chemical recycling of waste PET into crumb rubber modified asphalt. Journal of Cleaner Production, 180, 682-688. 


\section{Figure captions:}

Figure 1. Aggregate distribution with NMAS= $12.5 \mathrm{~mm}$

Figure 2. schematic of fracture energy to failure

Figure 3. Penetration Results of different asphalt binders

Figure 4. Softening point of different asphalt binders

Figure 5. rotational viscosity of modified binders

Figure 6. rutting parameter of base and modified asphalt binders: a) before aging b) after aging

Figure 7. Results of MSCR of binders

Figure 8. Results of resilient modulus of modified mixtures

Figure 9. ITS values of mixtures

Figure 10. Flow number values of mixtures

Figure 11. Rut depth values of mixtures

Figure 12. Fatigue life of mixtures

Figure 13. fracture energy of different mixtures 


\section{Table:}

Table 1 . aggregates properties

Table 2. aggregate's Chemical properties

Table 3. binder properties

Table 4. Properties of fibers

Table 5. Properties of crumb rubber

Table 6. Properties of PET

Table 7. Physical and chemical components of three types of ASAs.

Table 8. Sample Identification of modified bitumens

Table 9. MSCR test results

Table 10. VECD coefficients of different modified binders

Table 11. Fatigue life of different binders

Table 12. Asphalt binder grades based on PG and MSCR systems. 